\title{
DTI-Based Neuronavigation Guided Eloquent Area Glioma Resection with Awake Craniotomy: Supra-Functional Resection of Eloquent Area Gliomas
}

\author{
Ali AKAY ${ }^{1}$, Rauf NASIROV ${ }^{1}$, Mete RUKSEN ${ }^{1}$, Cenk ERASLAN ${ }^{2}$, Sertac ISLEKEL ${ }^{1}$ \\ ${ }^{1}$ Kent Hospital, Department of Neurosurgery, Izmir, Turkey \\ ${ }^{2}$ Ege University, School of Medicine, Department of Radiology, Izmir, Turkey \\ This study has been presented at the $32^{\text {nd }}$ Annual Scientific Congress of Turkish Neurosurgical Society between 20 and 24 April 2018 at Antalya, Turkey \\ and $18^{\text {th }}$ European Congress of Neurosurgery between 21 and 25 October 2018 at Brussels, Belgium.
}

Corresponding author: Ali AKAY dr.aliakay@gmail.com

\section{ABSTRACT}

AIM: To present an overview of our awake craniotomy practices performed with complementary use of diffusion tensor imaging (DTI)-based neuronavigation (DTI-bN) and cortical-subcortical electrical stimulation for glioma resection located in eloquent areas, and the clinical outcomes of these practices regarding neurological morbidity and residual tumour volume.

MATERIAL and METHODS: This study was conducted between October 2016 and December 2017 involving 18 cases. The DTIbN method, in addition to cortical-subcortical stimulation, was done with awake craniotomy. Changes in the neurological status of the patients and results of the method regarding residual tumour volume were recorded. This retrospective study use data obtained from patients' records and radiological examinations.

RESULTS: Of the 18 patients, a gross total resection was performed on nine patients (50\%), a near total resection was performed on seven patients $(38.8 \%)$ and a subtotal resection was performed on two patients $(11.1 \%)$. Intraoperative neurological deterioration was observed in 14 of 18 patients during their surgical procedures, and neurological examinations in the sixth post-operative month revealed permanent deficits in two patients.

CONCLUSION: This study is one of the rare series to have presented the results of the use DTI-bN method as well as corticalsubcortical stimulation during awake craniotomy, according to literature review. In $88 \%$ of cases, tumour resection rates are over $90 \%$.

KEYWORDS: Awake craniotomy, Cortical-subcortical stimulation, DTI-based neuronavigation, Eloquent area glioma, Suprafunctional

\section{INTRODUCTION}

$\mathrm{M}$ aximal surgical resection of gliomas is known to be the most effective method of prolonging the survival time of patients $(15,23,24)$. Several different terminologies have been used to define maximal resection, such as supra-marginal, supra-complete and supra-zonal resection, in all of which the radiological boundary is defined as the removal of contrasting tissue in high-grade tumours and the removal of hyperintense lesions on T2-weighted sections in low-grade gliomas $(7,11,14,21,30)$.

Supra-total resection was reported to have positive effect on the survival time by inhibiting anaplastic transformations in case of invasions that cannot be radiologically detected at the $20-\mathrm{mm}$ area of the tumour periphery (33). Supra-complete
Ali AKAY (iD) : 0000-0002-7187-398X

Rauf NASIROV (D) : 0000-0001-5192-6207
Mete RUKSEN (1) : 0000-0002-0521-8143

Cenk ERASLAN (1D) : 0000-0002-5762-6149
Sertac ISLEKEL (10) : 0000-0001-8091-1426 
resection in a single surgical session has become an ideal practice for gliomas with the introduction of intraoperative magnetic resonance imaging (iMRI), although it is not always possible to call it as 'a safe complete resection' for the excision of glial tumours in eloquent areas $(8,34)$. The use of iMRI for these tumours cannot assist surgeons in preserving functional structures while performing complete radiologic resections (6). Thus, currently, the awake craniotomy method is the optimum safe resection method in cases that involved eloquent areas. During awake craniotomy, other methods such as corticalsubcortical stimulation (CSC) and intraoperative neurophysiological monitoring are used to distinguish and map eloquent areas and allow safe resections, although complete resection cannot be achieved in eloquent area-localised gliomas $(5,19)$. Intraoperative neuronavigation, which has gained popularity as a key technology during surgical procedures, has been commonly used in glial tumour surgeries; however, it has potential for errors, e.g. brain shift $(9,12,20)$.

Thus, this study aimed to present an overview of our awake craniotomy practices performed with complementary use of diffusion tensor imaging (DTI)-based neuronavigation (DTI-bN) and CSC for glioma resection located in eloquent areas and to reveal the clinical outcomes of these practices in terms of neurological morbidity and residual tumour volume. Given the difference in other terms -supra-marginal, supra-complete and supra-total resection-used thus far in literature to define the extent of tumour resection, this study used the term 'suprafunctional resection' to define the maximal extent of glioma resection that evades permanent, irreversible functional deficits at the expense of temporary functional impairments in patients with eloquent area glioma during awake craniotomy.

\section{- MATERIAL and METHODS}

This study involved initially 20 patients operated on in our clinic between October 2016 and December 2017, with a diagnosis of glial tumour located in, or close to, the eloquent area. During the awake craniotomy, Mayfield skull pin fixation was used together with CSC to prevent DTI-bN-tract damage. Given the deviations in the navigation system due to poor input data quality during surgical operations, two patients were excluded from this study. The post-operative neurological status of all patients was recorded at post-operative day 1 , week 1 , month 3 and month 6 and retrospectively studied to present the results of tumour resections and the residual tumour volume of the eloquent area-located lesions.

\section{Radiology}

\section{Pre-operative radiological examination}

Contrast- and non-contrast-enhanced MRIs and functional and DTI examinations were made with $1 \mathrm{~mm}$ sections on the day before the operation. Pre-operative MR images (T2weighted and contrast-enhanced T1 imaging) and DTI-MR images were fused on the S7 Medtronic Stealth Station and used as guides during the excision (Figure 1A).

\section{Post-operative radiological examination}

All patients underwent a post-operative control cranial MRI within 72 hours (Figure 1D).
Results of the pre-operative and post-operative cranial MRI were examined, and residual tumour volumes were calculated by neuroradiologists. In these calculations, T2-weighted fluid attenuation inversion recovery images were used for lowgrade glioma and contrast-enhanced T1-weighted images were used for high-grade glioma to determine the tumour boundaries. The OsiriX MD software was used for the volumetric calculation.

\section{Surgical Procedure}

Following the placement of skull pin fixation and insertion of the urine catheter and the scalp block with mild sedation, the entire surgical procedure was performed while the patients were fully awake. A local anaesthetic agent was applied to the incision line after the optimum incision boundaries were drawn on the skin based on the navigation.

The awake craniotomy method which has been performed in our clinic with the guidance of mapping by CSC with skull pin fixation has been reported in detail in a previous article (1). Eloquent areas were mapped via cortical stimulation, and a safe cortical incision was made with a rigid DTI-bN (Figure 1G). The bipolar cautery used during the excision of the glial tumours was introduced into the navigation system and used as a navigation probe (Figure $1 \mathrm{H}$ ). During awake craniotomy, $50-80 \mathrm{~Hz}, 0.5 \mathrm{msec}$ biphasic waves were used for direct stimulation (Ojeman Cortical Stimulator, OCS2 Integra Neurosciences, Plainsboro, NJ). The electrical current was increased from $1 \mathrm{~mA}$ to $3-5 \mathrm{~mA}$ with $0.5 \mathrm{~mA}$ steps. White matter fibres (corticospinal tracts, arcuate fasciculus, uncinate fasciculus, superior longitudinal fasciculus, middle longitudinal fasciculus, inferior longitudinal fasciculus, frontal aslant tracts) adjacent to the lesion were identified in the intraoperative DTI-bN programme (Figure 1B, C, E, F). Tumour excision was initiated at the safe tumour boundaries without neighbouring tracts, and any neurological changes observed during surgical excisions were shared with the surgical team. When neurological deteriorations associated with the excision of lesions adjacent to tracts were detected, resections were stopped and interventions were directed accordingly (Figure 1I).

Deteriorations of speech and motor-sensory function during neurophysiological follow-up and tumour boundaries demonstrated by DTI-bN with a rigid pin fixation were parameters that determined the boundaries of the surgical excision.

\section{RESULTS}

This study involved 18 patients with functional lesions, including 15 male and three female patients with a median age of 46 (age range, 26-75) years. In all patients, the left hemisphere was dominant. Table I presents demographic characteristics, symptoms that led to the diagnosis, and radiologic and functional tumor locations.

\section{Intraoperative Neurological Deficits}

Neurological examination of patients revealed pre-operative neurological deficits in four of 18 patients involved in the study and no such deficits in the remaining 14 patients. Neurological 


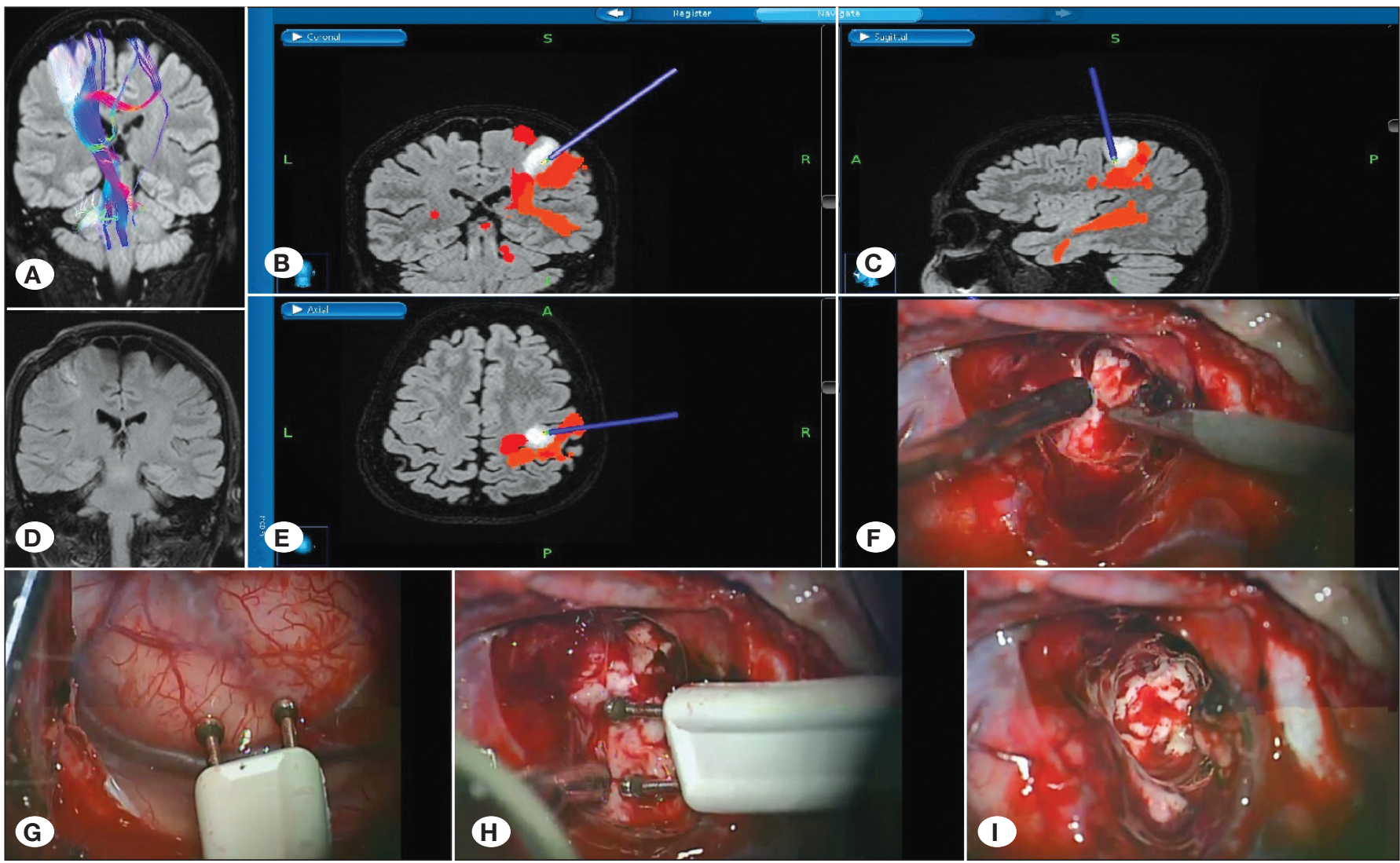

Figure 1: Precentral gyrus oligodendroglioma resection. A) Pre-operative diffusion tensor images showing relations between corticospinal tracts and glioma. Intraoperative neuronavigation images (B: coronal; C: sagittal) showing the position of the navigation probe with the resection border and corticospinal tracts. D) Post-operative fluid attenuation inversion recovery T1-weighted magnetic resonance imaging showing gross total resection. E) Axial intraoperative neuronavigation images showing the position of the navigation probe, resection border and corticospinal tracts. F) Simultaneous intraoperative microscope image of intraoperative navigation image shown in Figure 1E. G) Intraoperative microscope photography showing cortical stimulation by bipolar stimulation. H) Intraoperative microscope photography showing subcortical stimulation by bipolar stimulation. I) Intraoperative microscope photography showing cavity after tumour removal.

deterioration was recorded during surgeries of three of the four patients with a pre-operative deficit: worsening hemiplegia and aphasia (1), monoplegia (1) and severe hemiparesis (1), while no change was intraoperatively observed in the level of the neurological deficit in the remaining one patient.

Neurological deficits developed at various levels during surgery in 11 of these 14 patients, who had no pre-operative neurological deficits, i.e. hemiparesis (6), hemiparesis with aphasia (4) and monoparesis (1), while the remaining three patients maintained their neurological status.

\section{Intraoperative Complications}

None of the patients developed any complications such as vascular injury, post-operative bleeding or infection due to the surgical procedure. Although $1000 \mathrm{mg}$ of levetiracetam was infused intravenously for each patient during skin incision, intraoperative focal seizures were observed in three patients during CSC, i.e. focal seizure in the upper extremities in two patients and facial seizure in one patient. Patients with focal seizure in upper extremities developed Todd's paresis. The resection was terminated in one of the two patients having paresis, as the seizure occurred almost at the end of the tumour resection during the CSC in that patient. In the second patient with focal seizure in upper extremities, Todd's paresis developed after motor cortex mapping was completed, but with the assumption that the limits of tumour resection defined by DTI-bN were valid, the surgery continued and resection was completed. According to the post-operative residual tumour volume results, a near total tumour resection was performed in patient 1 , whereas a gross total resection (GTR) was achieved in patient 2.

\section{Surgical Resection Rates}

Of the 18 patients in this study, GTR was performed in nine patients $(50.0 \%)$, near total resection in seven patients $(38.9 \%)$ and subtotal resection in two patients $(11.1 \%)$ (Table II). The histopathological distribution of the gliomas were oligodendroglioma in six patients, glioblastoma (GBM) in five patients, anaplastic oligodendroglioma in three patients, astrocytomas in three patients and pilocytic astrocytomas in one patient. Table II presents numbers and rates of surgical resection according to glioma grades. 


\section{Post-Operative Neurological Follow-Up}

Intraoperative neurological deterioration was seen in 14 of 18 patients who underwent glioma resection. In nine of the 11 patients who had no pre-operative neurological deficits

Table I: Data of Patients Who Underwent Suprafunctional Resection (Demographic, Symptom, Functional, Radiological Site)

\begin{tabular}{|c|c|}
\hline Age, median (range) (years) & $46(26-75)$ \\
\hline Male / Female (n) & $15 / 3$ \\
\hline Symptoms leading to diagnosis & n (\%) \\
\hline Headache & $4(22.2)$ \\
\hline Epileptic seizure & $9(50)$ \\
\hline Sensory / motor deficit & $4(22.2)$ \\
\hline Congitive symptom & $1(5.6)$ \\
\hline Tumor Location; Functional & n (\%) \\
\hline Right precentral cortex & $4(22.2)$ \\
\hline Left precentral cortex & $5(27.8)$ \\
\hline Right postcentral cortex & $2(11.1)$ \\
\hline Speech area (broca) & $3(16.7)$ \\
\hline Right capsula interna & $2(11.1)$ \\
\hline Left capsula interna & $1(5.6)$ \\
\hline Left postcentral cortex & $1(5.6)$ \\
\hline Tumor Location; Radiological & n (\%) \\
\hline Right frontal & $6(33.3)$ \\
\hline Left frontal & $7(38.7)$ \\
\hline Right parietal-occipital & $1(5.6)$ \\
\hline Left temporal-insular & $1(5.6)$ \\
\hline Left fronto-temporal-insular & $1(5.6)$ \\
\hline Right fronto-bazal-temporal-insular & $1(5.6)$ \\
\hline Left temporol-occipital & $1(5.6)$ \\
\hline
\end{tabular}

but who had intraoperative neurological deterioration, the neurological deficits were observed to have recovered in postoperative month 1. The neurological deficit of one patient was seen recovered in post-operative month 3 . On the contrary, no recovery was observed in the neurological deficit-hemiparesis and aphasia-of the final patient at the post-operative month 6 . Compared to their pre-operative status, at the post-operative month 6 , recovery was observed in two of the three patients whose pre-operative neurological deficits were worsened intraoperatively. No recovery of neurological deficit was observed in one remaining patient, who had monoparesis in the lower extremities. Table III presents the neurological deficits seen in all patients involved in the current study, along with their pathological diagnoses and recovery times.

Of the nine patients in whom GTR was achieved, five patients were observed to develop intraoperative neurological deficits. The deficits in the four of these five patients recovered within 1 month after surgery, whereas the deficit of the remaining patient became permanent. The patient with permanent deficit was diagnosed with GBM-isocitrate dehydrogenase (IDH) wildtype. Intraoperative neurological deterioration was seen in six of the seven patients who underwent near total resection: neurological deterioration was restored at postoperative month 1 in four patients, and recovery in neurological deterioration was observed at post- operative month 3 and 6 in other two patients, without leaving any permanent deficits. Patients with late recovery were diagnosed with anaplastic oligodendroglioma-IDH mutant (former patient) and GBM-IDH mutant (later patient). Intraoperative deteriorations were seen in both patients who underwent subtotal resection; patient 1 achieved neurological recovery within the first month, while patient 2 still had permanent neurological deficit (Figure 2). Of the 18 patients, surgery-related deficits were observed to have become permanent in two patients $(11.1 \%)$ at the 6 -month follow-up. The patient with pre-operative neurological deficits was one of the two patients who retained their permanent neurological deficits at the 6-month post-operative followup. The follow-up period lasted for 6 months following awake craniotomy with an assumption that the post-operative rehabilitation programmes were completed at that time.

\section{DISCUSSION}

The awake craniotomy method is preferred for safe resection of tumours that are in close proximity to or within eloquent areas, and intraoperative stimulation, which is used during awake craniotomies, is the main tool for the mapping of

Table II: Numbers and Rates of Surgical Resection According to Glioma Grades

\begin{tabular}{cccc}
\hline $\begin{array}{c}\text { WHO glioma grade } \\
\text { (n) }\end{array}$ & $\begin{array}{c}\text { Grosstotal } \\
\text { Resection (50.0\%) }\end{array}$ & $\begin{array}{c}\text { Neartotal } \\
\text { Resection (38.9\%) }\end{array}$ & $\begin{array}{c}\text { Subtotal } \\
\text { Resection (11.1\%) }\end{array}$ \\
\hline I (1) & 1 & 0 & 0 \\
\hline II (8) & 3 & 5 & 0 \\
\hline III (4) & 3 & 1 & 0 \\
\hline IV (5) & 2 & 1 & 2 \\
\hline
\end{tabular}


Table III: Histopathologic Diagnoses of Cases and Per-Op / Intra-Op / Post-Op Neurological Status of Patients

\begin{tabular}{|c|c|c|c|c|c|c|c|}
\hline $\begin{array}{l}\text { WHO Glioma } \\
\text { Grade } \\
\text { (n) }\end{array}$ & 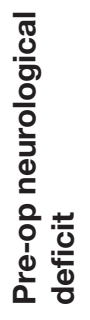 & 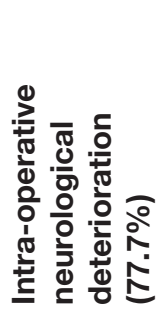 & 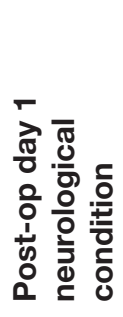 & 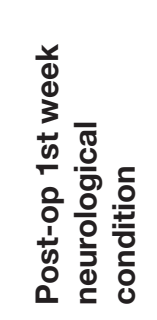 & 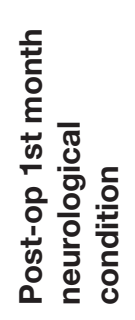 & 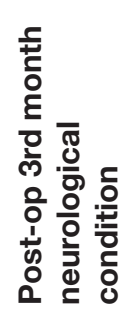 & 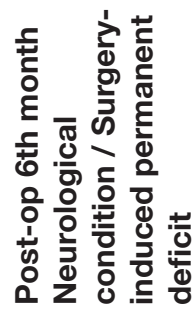 \\
\hline $\begin{array}{l}\mathrm{I} \rightarrow(1) \\
\quad \text { Pilocytic astrocytoma } \rightarrow(1)\end{array}$ & 0 & 0 & $1 \leftrightarrow$ & $1 \leftrightarrow$ & $1 \leftrightarrow$ & $1 \leftrightarrow$ & $1 \leftrightarrow / 0$ \\
\hline $\begin{array}{l}\text { II } \rightarrow \text { (8) } \\
\quad \text { Oligodendroglioma } \rightarrow(6) \\
\quad \text { Diffuse astrocytoma } \rightarrow(2)\end{array}$ & 0 & $2 \leftrightarrow / 6 \downarrow$ & $8 \leftrightarrow$ & $1 \uparrow / 4 \leftrightarrow / 3 \downarrow$ & $5 \uparrow / 3 \leftrightarrow$ & $8 \leftrightarrow$ & $8 \leftrightarrow / 0$ \\
\hline $\begin{array}{l}\text { III } \rightarrow \text { (4) } \\
\text { Anaplastic oligodendrogliom- } \\
\text { IDH mutant } \rightarrow(3) \\
\text { Anaplastic gemistocytic } \\
\text { astrocytoma } \rightarrow(1)\end{array}$ & 2 & $4 \downarrow$ & $2 \leftrightarrow / 2 \downarrow$ & $2 \uparrow / 2 \leftrightarrow$ & $3 \uparrow / 1 \leftrightarrow$ & $4 \leftrightarrow$ & $4 \leftrightarrow / 0$ \\
\hline $\begin{aligned} \text { GBM } & \rightarrow(5) \\
& \text { IDH wild } \rightarrow(4) \\
& \text { IDH mutant } \rightarrow(1)\end{aligned}$ & 2 & $1 \leftrightarrow / 4 \downarrow$ & $4 \leftrightarrow / 1 \downarrow$ & $1 \uparrow / 4 \leftrightarrow$ & $5 \leftrightarrow / 0$ & $1 \uparrow / 4 \leftrightarrow$ & $5 \leftrightarrow / 2$ \\
\hline
\end{tabular}

$\uparrow:$ Neurological deficit recovery, $\leftrightarrow:$ Neurological conditions table, $\downarrow$ : Neurological deficit increase, WHO: The 2016 World Health Organization Classification of Tumors of the Central Nervous System.

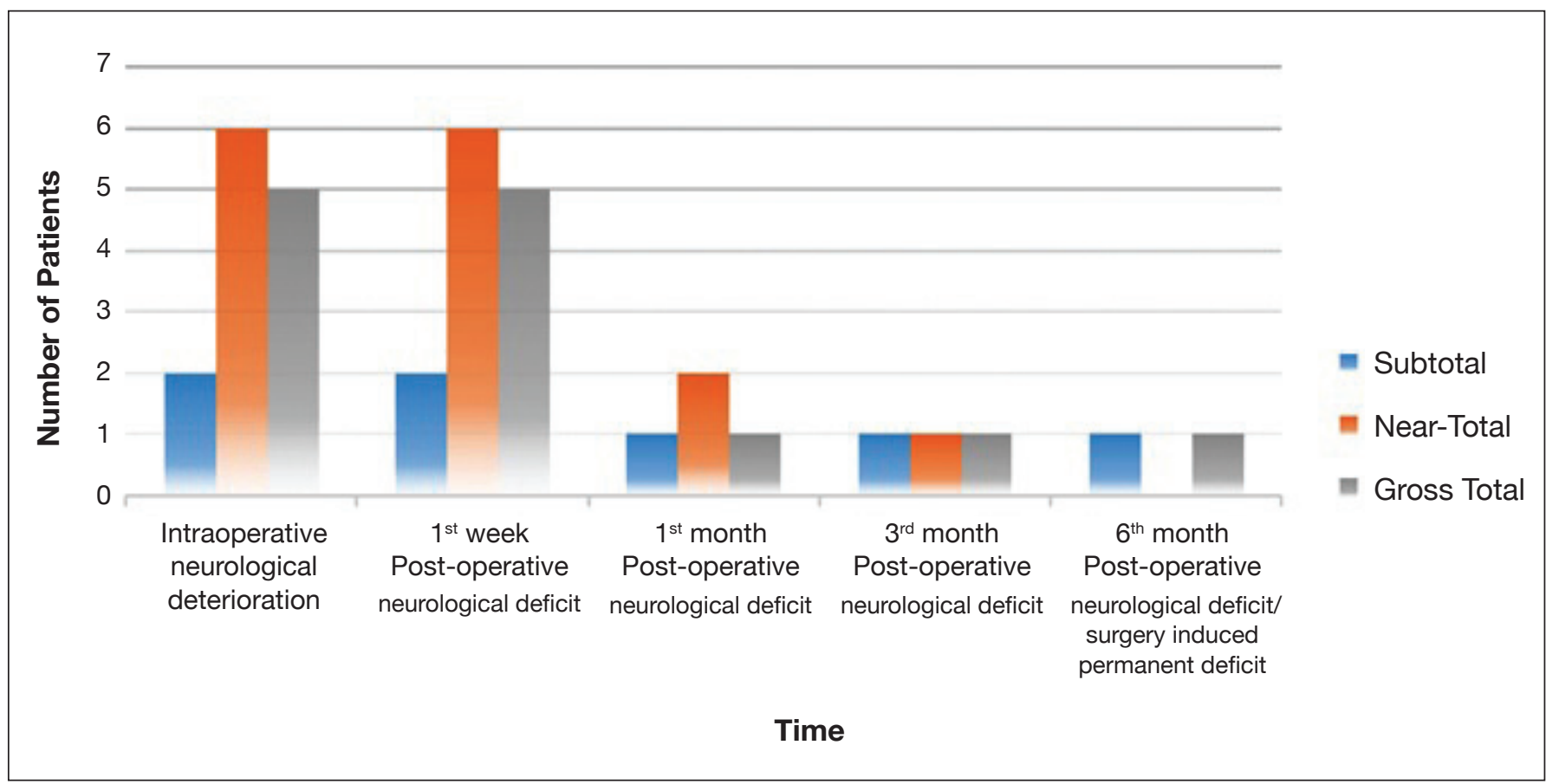

Figure 2: Distribution of the number of patients with neurological deficit according to glioma resection subgroups during follow-up. 
eloquent areas. As reported in a meta-analysis, permanent neurological deficits could be prevented by using CSC while the extent of tumour resection is increased particularly in cases with gliomas involving eloquent areas (4).

With this, it is hard to consider GTR or supra-total resection for tumours that involve eloquent areas despite the benefits of functional mapping for partial tumour resection. In this study, we report the use of DTI-bN for tumour resection in eloquent areas and its value for post-operative tumour volume and the occurrence of postoperatively persistent neurological deficits in patients who underwent awake craniotomy with functional mapping. A comparison of the frameless navigation system and frame-based stereotaxy reveals that both provided similar positional accuracy, with errors -usually being clinical or technical in nature-that occur during surgeries performed with neuronavigation $(18,20)$. Leaks of cerebrospinal fluid (CSF) during surgery and other volume-reducing activities such as decompression of cystic masses may lead to a brain shift and a shift of the tumour position, which invalidates the patient-to-image registration (29). Potential sources of technical problems can be listed as low-quality pre-operative surgery images and errors made during the identification of patient data (31). In this study, navigation-related deviations were detected in the pre-operative cranial MRls of two patients; therefore, those patients were excluded from this study. Additionally, the deviations were attributable to poor data input. In the presence of fast-growing neoplasms, data obtained days and weeks earlier should not be used during surgery. In several studies using data from iMRI examinations for neuronavigation systems, minimising errors that may occur when using such systems was suggested $(9,10,12)$. The images that become invalid because of brain shift due to CSF leak during surgery, or decompression of cystic lesions should be repeated with intraoperative MRI. Further studies have shown that iMRls contribute to the survival times of patients with gliomas $(3,16)$. In a study of Senft et al., iMRls have been reported to be superior to neuronavigation regarding complete resection during glioma surgery (26). However, iMRls is sometimes not practical to use, particularly when using the awake craniotomy method, as there may be problems with the patient being awake, body movements and increased operative duration $(13,26)$.

Moreover, not all operating rooms facilitate iMRls, which could limit the current experience in awake craniotomy practices. Considering all these, we used DTI-bN during awake craniotomies in this series to minimise errors of shifts that could occur due to intraoperative CSF loss and other volumereducing activities during glioma resection. In this way, errors related to brain shift were detected through subcortical stimulation, thus reducing the margin of error. While functional $\mathrm{MRI}$ reveals cortical functional areas, DTI shows the course of white matter fibre tracts. The use of images that include DTI-derived data in the neuronavigation also helps protect fibre tracts located adjacent to the tumour. The randomised controlled study of Wu et al. showed that DTI-bN reduces the rate of neurological deficits when compared to methods that involved navigation without DTI (32). However, in patients with brain shift, the position of fibre tracts changes with the positions of other brain structures. Currently, no system has accurately calculated this shift, and the use of subcortical electrical stimulation could an invaluable help to surgeons performing awake craniotomy. In a study conducted by Zolal et al., the actual localisation of the corticospinal region in 36 patients was predicted through subcortical electrical stimulation of the white matter during brain tumour resection, and a rough estimate of the true localisation of the corticospinal tract can be possible with DTI (35). In general, a response can be obtained by performing stimulation at an 8-mm distance from the corticospinal path predicted by DTI (13). However, the measured tumour-to-track distance with a positive stimulation was reported to be up to $15 \mathrm{~mm}$ in case of complementary use of intraoperative tractography and motor evoked potential (MEP) monitoring $(17,22)$. Additionally, conductivities of different brain structures and stimulation parametersare among those factors that affect the accuracy of subcortical mapping (2).

The study by Sollman et al., which was recently published as the first study in relevant literature, has presented the use of DTI-bN with direct electrical stimulation for the resection of highlylanguage eloquent gliomas as adjunct to awake craniotomy and analysed the clinical outcomes of this approach (28). The current study reports a series of patients with gliomas located in eloquent areas, and most gliomas were detected to be within and adjacent to the corticospinal tract. Additionally, this study presents the clinical outcomes of the adopted approach regarding the residual tumour volume and the occurrence of surgery-related neurological deficits.

The rate of stimulation-related epileptic seizure in awake craniotomy ranges from $3 \%$ to $20 \%(5,25,27)$. In the current series, the rate of intraoperative focal epilepsy was $13.3 \%$, although the stimulation current intensity for cortical and subcortical mapping varied from 3 to $5 \mathrm{~mA}$. No generalised epilepsy was observed during surgical interventions. Of two patients with seizure-induced paresis, the resection was discontinued in patient 1 , but it was continued in patient 2 since the motor cortex mapping had already been completed. The paresis of patient 1 , who had a subcortical stimulationinduced seizure during the resection, recovered within one week, while the paresis of patient 2 , which was caused by the seizure that developed during the motor cortex stimulation (motor mapping), recovered within 72 hours postoperatively. Post-operative $\mathrm{MRI}$ results of patient 2, who underwent resection within the boundaries defined by the DTI-bN, revealed that GTR was achieved despite the development of Todd's paresis during surgery.

Of the 18 patients included in this series, four patients were diagnosed with GBM-IDH wildtype and one patient was diagnosed with GBM-IDH mutant. Two of the four patients with GBM-IDH wildtype developed surgery-related deficits, and these neurological deficits were observed to have become permanent at the 6-month follow-up, which constitutes $11.1 \%$ of the patients involved.

There could be several potential reasons for the occurrence of persistent neurological deficits in patients with GBM-IDH wild type, i.e. plasticity due to rapid tumour progression 
did not occur, the size of the peritumoural oedema did not regress after the operation, and there were brain shifts during the excision of cystic and necrotic tumours tissues and damage to the peritumoural region and oedema occurring after brain radiotherapy. Thus, particularly, in high-grade gliomas adjacent to the precentral cortex, aggressive tumour excisions could have a negative effect on the median survival times by increasing the risk of permanent functional deficits. Intraoperative neurological deterioration was seen in six of the eight patients with grade II gliomas, and complete neurological recovery was observed in five patients within the first month and in one patient at the 6-month follow-up. All four patients with gliomas classified as grade III were observed to have intraoperative neurological deterioration, but neurological recovery was observed in three patients within the first month and in the other patient at the end of the sixth month. The neurological recovery rates of patients with grade II and III gliomas were similar. Permanent neurological deficits were not seen in these two patient groups. Of the 12 patients with gliomas (graded as II and III gliomas), GTR was possible in six patients, and a near total resection in the other six patients, and no permanent neurological deficits were identified in this group at the 6-month follow-up (Figure 3). The slow development of the glial tumours with grades II and III, the existence of plasticity and the non-existence of necrotic-cystic areas that may avoid brain shift during tumour excisions are thought to have a significant role in preventing permanent functional impairments caused by tumour excision in this series.
Based on our experience with combined use of DTI-bNof CSC in awake craniotomy, we believe this approach could have positive effects on survival times of patients and their having less surgery-related neurological deficits, particularly when applied to grade II and III functional area gliomas. Long-term follow-ups in future studies will clearly elucidate the effects of this approach on survival rates.

\section{Study Limitations}

Despite being one of the rare series reported so far on eloquent area glioma resection with complementary use of DTI-bN and CSC with awake craniotomy, this study has some limitations. First, due to the small patient population, there is no 'standalone CSC use with awake craniotomy' group of patients as a control group. Moreover, as stated in the discussion, forming homogeneous experimental and control groups that have the same characteristics in terms of plasticity of the functional area, glioma grade, distance between glioma and functional area could not be possible in a controlled trial with such a limited patient population. Thus, larger studies with a controlled design, which ensures that any difference found between the groups will be independent of any biasby blinding the patients, investigators and assessors-are required to present findings that will discuss the effectiveness of the aforementioned approach for eloquent area-localised glioma resection.

\section{CONCLUSION}

In this study, the term 'supra-functional resection' has been used to define the maximal extent of glioma resection that

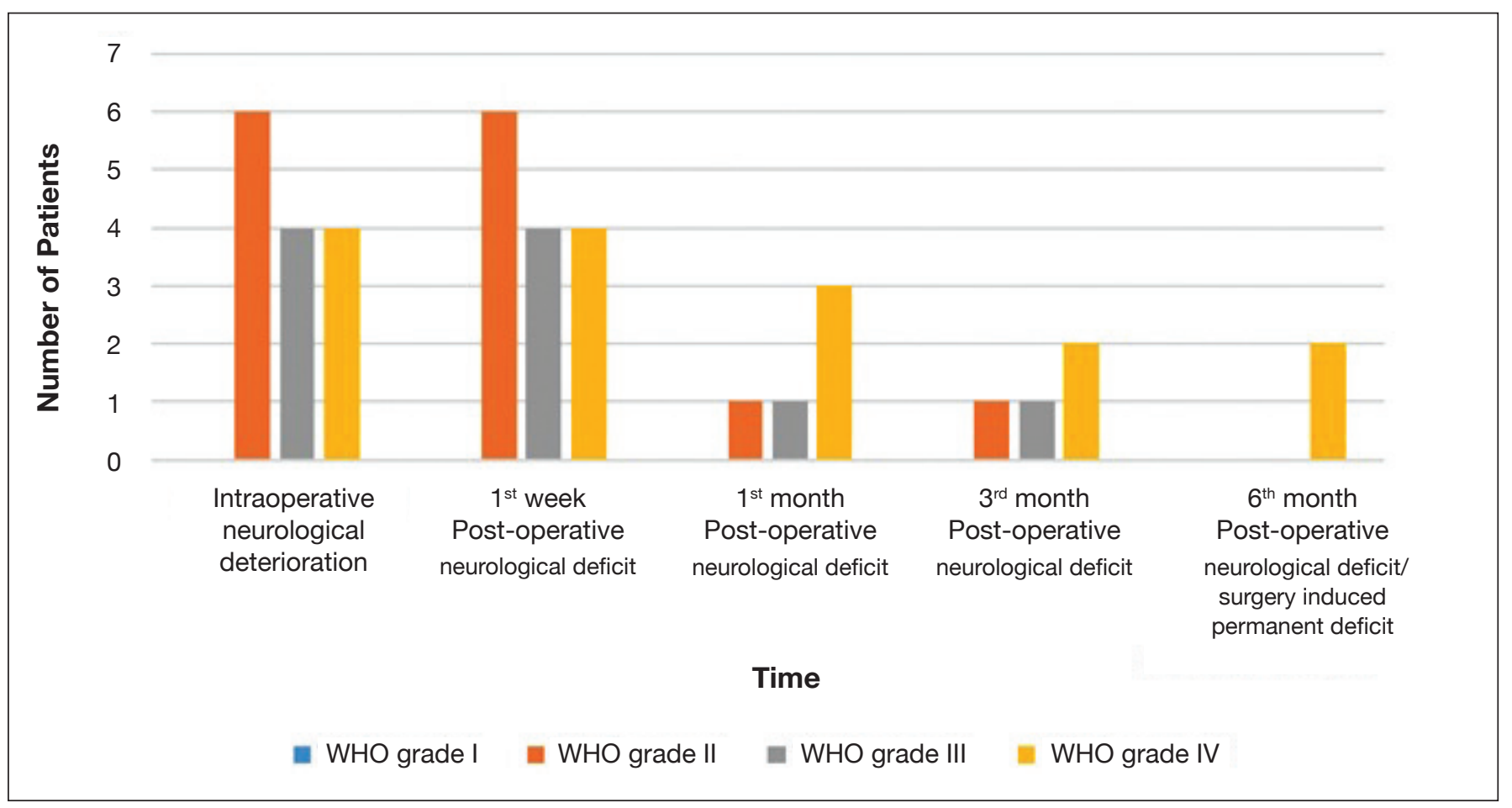

Figure 3: Distribution of the number of patients with neurological deficit according to the World Health Organization grade glioma classification during follow-up. 
evades permanent, irreversible neurological deficits at the expense of temporary functional impairments in patients. The combined use of DTI-bNand cortical-subcortical electrical stimulation for these tumours could help preserve functional areas during eloquent area glioma resection, while the damage to white matter fibre tracts and to cortical structures due to brain shift could be kept at a minimum degree with the help of this approach.

Therefore, further studies are needed on multimodal neuroimaging techniques for eloquent area glioma surgery in the setting of awake craniotomy, which will integrate neuronavigationalinformation into the operative workflow to prevent any navigational errors that may lead to permanent functional deficits and provide surgeons with the opportunity for maximised EOR and minimised the risk for surgery-related neurological morbidity.

\section{- REFERENCES}

1. Akay A, Islekel S: Awake craniotomy procedure: Its effects on neurological morbidity and recommendations. Turk Neurosurg 28(2):186-192, 2018

2. Boetto J, Bertram L, Moulinié G, Herbet G, Moritz-Gasser S, Duffau $\mathrm{H}$ : Electrocorticography is not necessary during Awake Brain Surgery for Gliomas. World Neurosurg 91:656,657, 2016

3. Claus EB, Horlacher A, Hsu L, Schwartz RB, Dello-lacono D, Talos F, Jolesz FA, Black PM: Survival rates in patients with low-grade glioma after intraoperative magnetic resonance image guidance. Cancer 103(6):1227-1233, 2005

4. De Witt Hamer PC, Robles SG, Zwinderman AH, Duffau $H$, Berger MS: Impact of intraoperative stimulation brain mapping on glioma surgery outcome: A meta-analysis. Clin Oncol 30(20):2559-2565, 2012

5. Duffau H: Contribution of cortical and subcortical electrostimulation in brain glioma surgery: Methodological and functional considerations. Neurophysiol Clin 37:373-382, 2007

6. Duffau $\mathrm{H}$ : The "frontal syndrome" revisited: Lessons from electrostimulation mapping studies. Cortex 48:120-131, 2012

7. Eyupoglu IY, Bauchfelder M, Savaskan NE: Surgical resection of malignant gliomas-role in optimizing patient outcome. Nat Rev Neurol 9:141-151, 2013

8. Eyupoglu IY, Hore N, Merkel A, Buslei R, Buchfelder M, Savaskan N: Supra-complete surgery via dual intraoperative visualization approach (diva) prolongs patient survival in glioblastoma. Oncotarget 7:25755-25768, 2016

9. Ferrant M, Nabavi A, Macq B, Black PM, Jolesz FA, Kikinis R, Warfield SK: Serial registration of intraoperative MR images of the brain. Med Image Anal 6(4):337-359, 2002

10. Ferrant M, Nabavi A, Macq B, Jolesz FA, Kikinis R, Warfield SK: Registration of 3-D intraoperative MR images of the brain using a finite-element biomechanical model IEEE Trans Med Imaging 20(12):1384-1397, 2001

11. Ganslandt O, Stadlbauer A, Fahlbusch R, Kamada K, Buslei R, Blumcke I, Moser E, Nimsky C: Proton magnetic resonance spectroscopic imaging integrated into image-guided surgery: Correlation to standard magnetic resonance imaging and tumor cell density. Neurosurgery 56:291-298, 2005
12. Hartkens T, Hill DL, Castellano-Smith AD, Hawkes DJ, Maurer CR Jr, Martin AJ, Hall WA, Liu H, Truwit CL: Measurement and analysis of brain deformation during neurosurgery IEEE Trans Med Imaging 22(1):82-92, 2003

13. Hirschberg H, Samset E, Hol PK, Tillung T, Lote K: Impact of intraoperative MRI on the surgical results for high-grade gliomas. Minim Invasive Neurosurg 48(2):77-84, 2005

14. Kamp MA, Dibue M, Niemann L, Reichelt DC, Felsberg J, Steiger HJ, Rapp M, Slotty PJ, Sabel M: Proof of principle: Supramarginal resection of cerebral metastases in eloquent brain areas. Acta Neurochir 154:1981-1986, 2012

15. Keles GE, Lamborn KR, Berger MS: Low-grade hemispheric gliomas in adults: A critical review of extent of resection as a factor influencing outcome. J Neurosurg 95:735-745, 2001

16. Kubben PL, terMeulen KJ, Schijns OE, terLaak-Poort MP, van Overbeeke JJ, van Santbrink H: Intraoperative MRI-guided resection of glioblastoma multiforme: A systematic review. Lancet Oncol 12(11):1062-1070, 2011

17. Maesawa S, Fujii M, Nakahara N, Watanabe T, Wakabayashi $\mathrm{T}$, Yoshida J: Intraoperative tractography and motor evoked potential (MEP) monitoring in surgery for gliomas around the corticospinal tract. World Neurosurg 74(1):153-161, 2010

18. Mascott CR: In vivo accuracy of image guidance performed using optical tracking and optimized registration. J Neurosurg 105(4):561-567, 2006

19. Morsy AA, Ng WH: Awake craniotomy using electromagnetic navigation technology without rigid pin fixation. $J$ Clin Neurosci 22(11):1827-1829, 2015

20. Orringer DA, Golby A, Jolesz F: Neuronavigation in the surgical management of brain tumors: Current and future trends. Expert Rev Med Devices 9(5):491-500, 2012

21. Persano L, Rampazzo E, Della Puppa A, Pistollato F, Basso G: The three-layer concentric model of glioblastoma: Cancer stem cells, microenvironmental regulation, and therapeutic implications. Scientific World Journal 11:1829-1841, 2011

22. Prabhu SS, Gasco J, Tummala S, Weinberg JS, Rao G: Intraoperative magnetic resonance imaging- guided tractography with integrated monopolar subcortical functional mapping for resection of brain tumors. Clinical Article $\mathrm{J}$ Neurosurg 114(3):719-726, 2011

23. Sanai N, Berger MS: Glioma extent of resection and its impact on patient out- come. Neurosurgery 62:753-766,2008

24. Sanai N, Chang S, Berger MS: Low-grade gliomas in adults. J Neurosurg 115: 948-965,2011

25. Sartorius CJ, Berger MS: Rapid termination of intraoperative stimulation-evoked seizures with application of cold Ringer's lactate to the cortex. Technical note. J Neurosurg 88:349-351, 1998

26. Senft C, Bink A, Franz K, Vatter H, Gasser T, Seifert V: Intraoperative MRI guidance and extent of resection in glioma surgery: A randomised, controlled trial. Lancet Oncol 12(11):997-1003, 2011

27. Serletis D, Bernstein M: Prospective study of awake craniotomy used rou- tinely and nonselectively for supratentorial tumors. J Neurosurg 107:1-6, 2007 
28. Sollmann N, Kelm A, Ille S, Schröder A, Zimmer C, Ringel F, Meyer B, Krieg SM: Setup presentation and clinical outcome analysis of treating highly language-eloquent gliomas via preoperative navigated transcranial magnetic stimulation and tractography. Neurosurg Focus 44(6):E2, 2018

29. Spetzger $U$, Hubbe $U$, Struffert $T$, Reinges $M H$, Krings $T$, Krombach GA, Zentner J, Gilsbach JM, Stiehl HS: Error analysis in cranial neuronavigation. Minim Invasive Neurosurg 45(1):6-10, 2002

30. Stadlbauer A, Bauchfelder M, Doelken MT, Hammen T, Ganslandt O: Magnetic resonance spectroscopic imaging for visualization of the infiltration zone of glioma. Cent Eur Neurosurg 72:63-69, 2011

31. Widmann G, Schullian P, Ortler M, Bale R: Frameless stereotactic targeting devices: Technical features, targeting errors and clinical results. Int J Med Robot 8(1):1-16, 2018
32. Wu JS, Zhou LF, Tang WJ, Mao Y, Hu J, Song YY, Hong $\mathrm{XN}$, Du GH: Clinical evaluation and follow-up outcome of diffusion tensor imaging-based functional neuronavigation: A prospective, controlled study in patients with gliomas involving pyramidal tracts. Neurosurgery 61(5):935-948, 2007

33. Yordanova Y, Moritz-Gasser S, Duffau H: Awake surgery for WHO grade II gliomas within "non-eloquent" areas in the left dominant hemisphere: Toward a "supra-total" resection. J Neurosurg 115:232-239, 2011

34. Zhuang DX, Wu JS, Yao CJ, Qiu TM, Lu JF, Zhu FP, Xu G, Zhu W, Zhou LF: Intraoperative multi-information-guided resection of dominant-sided insular gliomas in a 3-T intraoperative magnetic resonance imaging integrated neurosurgical suite. World Neurosurg 89:84-92, 2016

35. Zolal A, Hejcl A, Vachata P, Bartoš R, Humhej I, Malucelli A, Nováková $M$, Hrach K, Derner $M$, Sameš $M$ : The use of diffusion tensor images of the corticospinal tract in intrinsic brain tumor surgery: A comparison with direct subcortical stimulation. Neurosurgery 71(2):331-340, 2012 